\title{
Analysis of Tilt-Buckling of Euler Columns with Varying Flexural Stiffness Using Homotopy Perturbation Method
}

\author{
S.B. Coşkun
}

Kocaeli University, Faculty of Engineering, Department of Civil Engineering Umuttepe Campus, 41380, Kocaeli, Turkey

E-mail: sb.coskun@kocaeli.edu.tr

Received June 2, 2009; revised December 14, 2009; published online July 15, 2010

\begin{abstract}
In this paper, the Homotopy Perturbation Method (HPM), is introduced for elastic stability analysis of tilt-buckled columns with variable flexural stiffness. Buckling loads and corresponding mode shapes are determined considering different types of variations in flexural stiffness of columns. The proposed approach is an efficient technique for the elastic stability analysis of specified problems.
\end{abstract}

Keywords: homotopy perturbation method, tilt-buckling, Euler column, elastic stability.

AMS Subject Classification: 34B15; 76A10.

\section{Introduction}

In most of the real world engineering applications, stability analysis of the compressed members is very important. There have been lots of researches related to the buckling behaviour of the axially compressed members. On the other hand, finding a closed-form solution for the buckling of elastic columns with variable cross-section subjected to complicated load configurations is very difficult in most of the cases.

The problems of buckling of columns under variably distributed axial loads were solved in detail by Vaziri and Xie [30] and others. Some analytical closedform solutions are given by Dinnik [8], Karman and Biot [14], Morley [20], Timoshenko and Gere [29] and others. One of the detailed reference related to the structural stability topic is written by Simitses and Hodges [26] with detailed discussions. Iyengar [21] made some analysis on buckling of uniform beam with several elastic support. Wang et al. [31] gave exact solutions for buckling of structural members for various cases of columns, beams, arches, rings, plates and shells. Ermopoulos [9] found the solution for buckling of tapered bars, axially compressed by concentrated loads applied at various locations 
along their axes. Li [18] gave the exact solution for buckling of non-uniform columns under axial concentrated and distributed loading. Lee and Kuo [17] established an analytical procedure to investigate the elastic stability of a column with elastic supports at the ends under uniformly distributed follower forces. Furthermore, Gere and Carter [10] investigated and established exact analytical solutions for buckling of several special types of tapered columns with simple boundary conditions. Solution of the problem of the buckling of elastic columns with step varying thickness is established by Arbabei and Li [1]. Stability problems of uniform bar with several elastic supports using the moment-distribution method were analyzed by Kerekes [16]. Research of Siginer [25] was about the stability of a column, which flexural stiffness has a continuous linear variation along the column. Moreover, the analytical solutions of a multi-step bar with varying cross section were obtained by Li et al. $[18,19]$ and papers cited therein. The energy method was used by Sampaio et al. [22] to find the solution for the problem of buckling behaviour of inclined beam-column. Some of the important researchers who studied the mechanical behavior of beams/columns are Keller [15], Tadjbakhsh and Keller [27] and Taylor [28]. Atanackovic [2] examined the stability problems and equilibrium states of elastic rods in detail with Euler energy and dynamical methods for a number of problems. Coşkun and Atay [7] and Atay and Coşkun [4] studied column buckling problems for the columns with variable flexural stiffness and for the columns on elastic foundation by using variational iteration method.

Homotopy Perturbation Method (HPM) is a coupling of the traditional perturbation method and homotopy in topology was proposed and improved by He $[11,12,13]$. The method was applied to nonlinear oscillators with discontinuities, nonlinear wave equations, boundary value problems, solid and fluid mechanics [23, 24]. Atay [3] and Coşkun [6] successfully applied the method for the determination of critical buckling loads for variable stiffness Euler columns, and Euler columns of variable flexural stiffness on an elastic foundation. These studies showed that the HPM was capable of analyzing elastic stability problems of Euler columns with variable stiffness.

In this study, homotopy perturbation method is introduced for the analysis of tilt-buckling of variable stiffness Euler columns. By the use of the method, an approximate continuous polynomial function is obtained as the solution for the problem at hand for which an analytical solution is not available. Once the buckling loads are obtained, corresponding modeshapes can be produced easily. The study presents an easy-to-use and efficient solution algorithm for the problems for which an analytical solution is difficult to obtain.

\section{Tilt Buckling of Columns}

Simitsis and Hodges [26] discussed tilt-buckling of columns and shafts for Euler columns with constant flexural stiffness theoretically. A tilt-buckled column is shown in Fig. 1. The governing equation and associated boundary conditions 


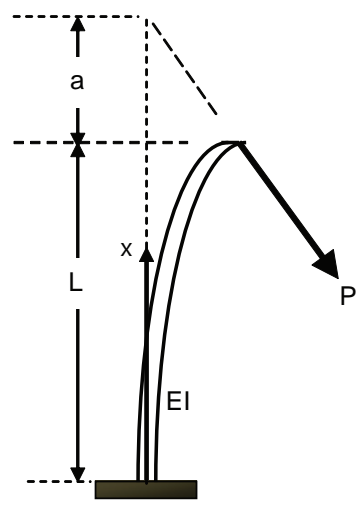

Figure 1. Tilt-buckled column.

for such a column are given as follows:

$$
\begin{aligned}
& \left(E I w_{, x x}\right)_{, x x}+P w_{, x x}=0, \\
& w(0)=0, \quad w_{, x}(0)=0, \\
& w_{, x x}(L)=0, \quad-\left[E I w_{, x x x}(L)+P w_{, x}(L)\right]=-P\left[-\frac{w(L)}{a}\right] .
\end{aligned}
$$

Solution of Eq. (2.1) is given as

$$
w(x)=C_{1} \sin \lambda x+C_{2} \cos \lambda x+C_{3} x+C_{4},
$$

where $\lambda^{2}=P /(E I)$. As it was discussed in [26], using boundary conditions we get a characteristic equation and formulate the following stability criterion

$$
\tan \lambda L=\lambda L(1+a / L) .
$$

\section{Homotopy Perturbation Method}

In recent years, Homotopy Perturbation Method (HPM) can be considered as one of the most promising methods for nonlinear problems. It provides an analytical approximate solution for problems at hand. Brief theoretical steps can be given as follows. Let consider a problem

$$
L(w)+N(w)=f(r), \quad r \in \Omega
$$

with boundary conditions $B(w, \partial w / \partial n)=0$. Here $L$ is a linear operator, $N$ is a nonlinear operator, $B$ is a boundary operator, and $f(r)$ is a known analytic function. HPM, which was described in detail in [13], defines homotopy as

$$
v(r, p)=\Omega \times[0,1] \rightarrow R,
$$

which satisfies the following equalities:

$$
H(v, p)=(1-p)\left[L(v)-L\left(w_{0}\right)\right]+p[L(v)+N(v)-f(r)]=0
$$


or

$$
H(v, p)=L(v)-L\left(w_{0}\right)+p L\left(w_{0}\right)+p[N(v)-f(r)]=0,
$$

where $r \in \Omega, p \in[0,1]$ is an imbedding parameter and $u_{0}$ is an initial approximation which satisfies the boundary conditions. Obviously, from (3.3) and (3.4), we have:

$$
\begin{aligned}
& H(v, 0)=L(v)-L\left(w_{0}\right)=0, \\
& H(v, 1)=L(v)+N(v)-f(r)=0 .
\end{aligned}
$$

As $p$ changes from zero to unity, $v(r, p)$ changes from $u_{0}$ to $u(r)$. In topology, these deformations $L(v)-L\left(w_{0}\right)$ and $L(v)+N(v)-f(r)$ are called homotopic. The basic assumption is that the solutions of Eq. (3.3) and Eq. (3.4) can be expressed as a power series in $p$ such that:

$$
v=v_{0}+p v_{1}+p^{2} v_{2}+p^{3} v_{3}+\ldots
$$

The approximate solution of $L(w)+N(w)=f(r), r \in \Omega$ can be obtained as:

$$
w=\lim _{p \rightarrow 1} v=v_{0}+v_{1}+v_{2}+v_{3}+\ldots
$$

The convergence of the series in Eq.(3.6) has been proved in [11]. Another study with important results on the convergence of HPM for partial differential equations was presented by Biazar and Ghazvini [5]. These studies proved the convergence of the method and showed that HPM is a reliable and efficient technique for analyzing nonlinear problems.

General buckling equation in (2.1) can be rewritten as follows,

$$
w_{, x x x x}+2 \frac{E I(x)_{, x}}{E I(x)} w_{, x x x}+\frac{1}{E I(x)}\left[P+E I(x)_{, x x}\right] w_{, x x}=0 .
$$

Based on the formulation given above, Eq. (3.7) can be divided into two parts as follows,

$$
L(w)=w_{, x x x x}, \quad N(w)=2 \frac{E I(x)_{, x}}{E I(x)} w_{, x x x}+\frac{1}{E I(x)}\left[P+E I(x)_{, x x}\right] w_{, x x}
$$

with $f(r)=0$. Inserting (3.5) into (3.4) in view of (3.7)-(3.8), and equating the like powers of the parameter $p$ and, then solving each equation yields the following iteration procedure

$$
\begin{aligned}
& p^{0}: \quad v_{0}^{i v}-w_{0}^{i v}=0, \\
& p^{1}: \quad v_{1}^{i v}+w_{0}^{i v}+\xi_{1}(x) v_{0}^{\prime \prime \prime}+\xi_{2}(P, x) v_{0}^{\prime \prime}=0 \\
& p^{2}: \quad v_{2}^{i v}+\xi_{1}(x) v_{1}^{\prime \prime \prime}+\xi_{2}(P, x) v_{1}^{\prime \prime}=0 \\
& p^{3}: \quad v_{3}^{i v}+\xi_{1}(x) v_{2}^{\prime \prime \prime}+\xi_{2}(P, x) v_{2}^{\prime \prime}=0 \\
& p^{n}: \quad v_{n}^{i v}+\xi_{1}(x) v_{n-1}^{\prime \prime \prime}+\xi_{2}(P, x) v_{n-1}^{\prime \prime}=0
\end{aligned}
$$


where

$$
\xi_{1}(x)=2 \frac{E I(x)_{, x}}{E I(x)}, \quad \xi_{2}(P, x)=\frac{1}{E I(x)}\left[P+E I(x)_{, x x}\right] .
$$

In order to apply HPM, a good initial guess is required and the solution of $L w=0$ provides a good one for the problem at hand. Inserting this initial guess into Eq.(3.9), a solution can easily be obtained by solving successive equations (3.9)-(3.14) and then using (3.6).

\section{Analysis of Tilt-Buckling of Columns Using HPM}

There exist four boundary conditions, i.e., two at each end of the column, in the problem under consideration. In previous works $[3,4,6,7]$, an initial approximation was chosen as the solution of the elastic stability problems of Euler columns. In the variational iteration method an initial approximation is chosen as the solution of the linear part of governing equation, i.e., solution of $L w=0$. Hence, a third order polynomial which is the solution of $L w=0$ for Eq. (3.7), was previously used in variational iteration analysis of buckling of Euler columns [7, 4]. The same initial approximation was also used in previous works employing HPM $[3,6]$ and is given as follows:

$$
w_{0}(x)=A x^{3}+B x^{2}+C x+D .
$$

This initial guess is a third order polynomial and has four unknown coefficients. These coefficients can be determined by introducing four boundary conditions at the column's ends into (4.1).

This polynomial function is also the interpolation function for the EulerBernoulli beam finite element. Variational form for this finite element requires an interpolation function to be continuous up to second order derivative. Such a function satisfies the essential boundary conditions for the element.

Since the initial approximation in Eq. (4.1) satisfies the essential boundary conditions at the column's ends, a simpler initial approximation may be chosen, satisfying $L w=0$ and the essential boundary conditions at $x=0$ automatically for the problem at hand. Such an approximation is given as:

$$
w_{0}(x)=A x^{3}+B x^{2} .
$$

This approximation is still a third order polynomial and includes only two unknown coefficients which are supposed to be found by imposing the rest two boundary conditions of the problem considered.

In the computations, fifteen iterations are conducted and boundary conditions for each case are rewritten by using the final approximation of iteration. Each boundary condition produces an equation containing two unknowns coming from the initial approximation. Hence two equations may be written with respect to those boundary conditions of the problem. These equations can be put into matrix form as follows:

$$
[M(\lambda)]\{A\}=\{0\}
$$


where $\{A\}=\langle A, B\rangle^{T}$. For a nontrivial solution, determinant of coefficient matrix must be zero. Determinant of matrix $[M(\lambda)]$ yields a characteristic equation in terms of $\lambda$. Positive real roots of this equation are the non-dimensional buckling loads for the tilt-buckled column.

\section{$5 \quad$ Case Studies}

\subsection{Column with constant flexural stiffness}

As the first case, tilt-buckling of an Euler column with constant flexural stiffness is considered for $a / L=0.8$. Stability criteria for this case is given in (7) [26]. By using the proposed HPM, fifteen iterations are conducted and the obtained results on the first three modes as non-dimensional buckling loads $\left(\lambda^{2}=P L^{2} / E I\right)$ are presented in Table 1 .

Table 1. Normalized buckling loads for the first three modes for the columns with a constant flexural stiffness $(a / L=0.8)$.

\begin{tabular}{ccc}
\hline MOD & HPM & Exact \\
\hline 1 & 1.2208 & 1.2208 \\
2 & 21.0864 & 21.0864 \\
3 & 60.5707 & 60.5707 \\
\hline
\end{tabular}

In addition to results given in Table 1, the errors at different iterations are depicted in Fig. 2 in order to show how fast the results are converging for each mode.

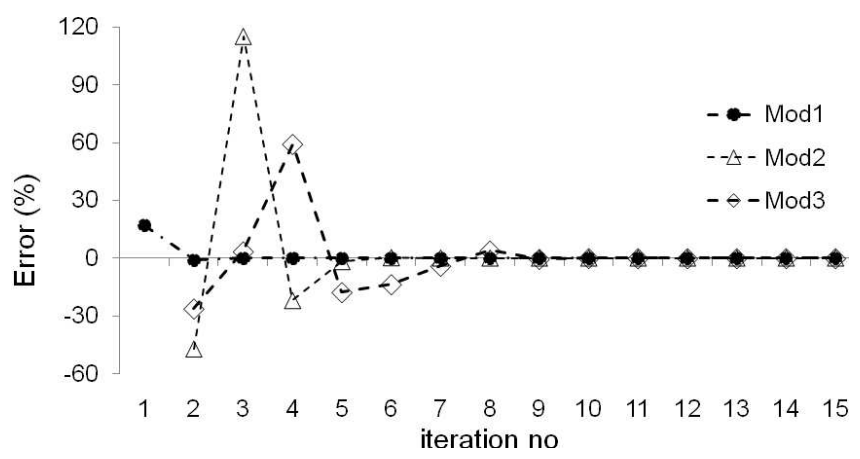

Figure 2. Variation of errors with iterations for the first three modes.

Variation of critical buckling loads with $a / L$ values is also considered and both HPM and exact results are compared in Fig. 3.

As follows from Table 1, results obtained by HPM are in excellent agreement with the exact results. Critical buckling load for constant stiffness case is converging very quickly as shown in Fig. 2. It follows from Fig. 3 that variations of critical buckling loads computed by HPM for different $a / L$ are the same as exact results. Comparisons with the exact solutions for constant stiffness case 


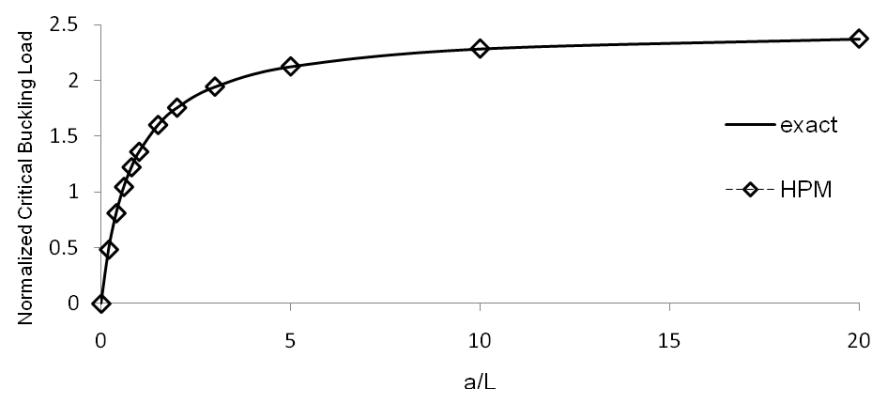

Figure 3. Variation of normalized critical buckling loads with $a / L$ values.

show that HPM is a powerful tool in predicting buckling loads of tilt-buckled columns. Hence, buckling loads for tilt-buckled columns with variable flexural stiffness can be produced by using HPM accordingly.

\subsection{Columns with variable flexural stiffness}

First, a column with an exponentially varied flexural stiffness is considered. Flexural stiffness of such a column can be considered as follows:

$$
\operatorname{EI}(x)=\alpha e^{-b x}
$$

The normalized buckling loads $\left(P L^{2} / \alpha\right)$ for the first three modes are computed for three different exponential variation cases: case $1(b L=0.1)$, case $2(b L=$ $0.5)$ and case $3(b L=1.0)$. As the second type of variation, a linearly varied flexural stiffness is considered. Linear variation of stiffness may be expressed as follows:

$$
\mathrm{EI}(x)=\alpha(1-b x)
$$

The normalized buckling loads for the first three modes are computed for three different linear variation cases: case $1(b L=0.1)$, case $2(b L=0.3)$ and case $3(b L=0.5)$. Finally, a quadratic variation of stiffness is considered. The variation is considered as follows:

$$
\mathrm{EI}(x)=\alpha(1-b x)^{2}
$$

For this quadratic variation case, normalized buckling loads for the first three modes are computed for three different quadratic variation cases: case $1(b L=$ $0.1)$, case $2(b L=0.3)$ and case $3(b L=0.5)$.

The variations in flexural stiffness defined by (5.1)-(5.3) are inserted in HPM formulation of the problem and buckling loads for the first three mode of tilt-buckled column are obtained, as shown in Table 2. Three cases in the table correspond to different values of parameters $a$ and $b$ as defined previously. Moreover, variations of critical buckling loads for different variable stiffness cases are depicted in Fig. 4. 
Table 2. Normalized buckling loads for first three modes for the columns with variable flexural stiffness $(a / L=0.8)$.

\begin{tabular}{lllll}
\hline Stiffness Variation & MOD & Case 1 & Case 2 & Case 3 \\
\hline \multirow{2}{*}{ Exponential } & 1 & 1.1878 & 1.0595 & 0.9083 \\
& 2 & 20.0754 & 16.4287 & 12.6791 \\
& 3 & 57.6260 & 47.0187 & 36.1338 \\
\hline \multirow{2}{*}{ Linear } & 1 & 1.1871 & 1.1159 & 1.0379 \\
& 2 & 20.0413 & 17.8579 & 15.5044 \\
& 3 & 57.5253 & 51.1559 & 44.2700 \\
Quadratic & 1 & 1.1537 & 1.0144 & 0.8652 \\
& 2 & 19.0395 & 15.0452 & 11.1789 \\
& 3 & 54.6080 & 42.9777 & 31.7185 \\
\hline
\end{tabular}

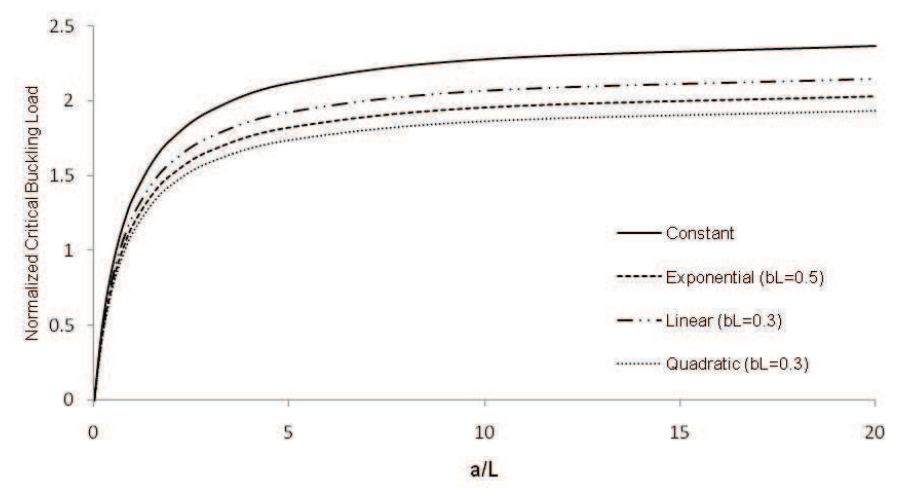

Figure 4. Variation of normalized critical buckling loads for different variable stiffness cases.

\section{Buckling Mode Shapes}

Buckling mode shapes for the columns may also be produced from the polynomial approximations by HPM considered in this study. For this purpose, buckling modes of constant flexural stiffness case obtained using HPM are compared with the exact ones given in [26]. Introducing eigenvalues into (2.1)-(2.3), we obtain eigenfunctions for the mode shapes:

$$
w(x)=A\left(\frac{5}{9 \lambda} \sin \lambda x-\cos \lambda x-\frac{5}{9} x+1\right) .
$$

Eigenfunctions in (2.3) are normalized and compared with the normalized polynomial eigenfunctions obtained by using HPM. These normalized eigenfunctions are defined as

$$
\bar{w}_{j}=\frac{w_{N}\left(x, \lambda_{j}\right)}{\left[\int_{0}^{1}\left|w_{N}\left(x, \lambda_{j}\right)\right|^{2} d x\right]^{1 / 2}}, \quad j=1,2,3, \ldots
$$


Figs. 5 and 6 show the comparison of buckling modes shapes for the first two modes. From these figures, excellent agreement between HPM and exact results is shown for both modes. Hence, the same approach can be used to predict buckling mode shapes for variable stiffness cases.

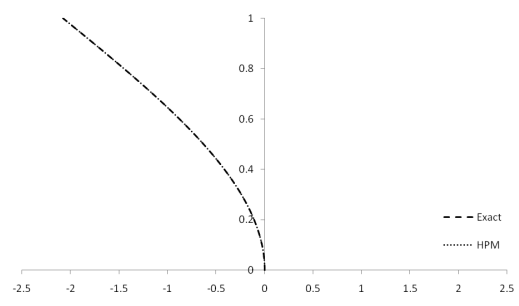

Figure 5. Mode shapes for the first mode - constant stiffness.

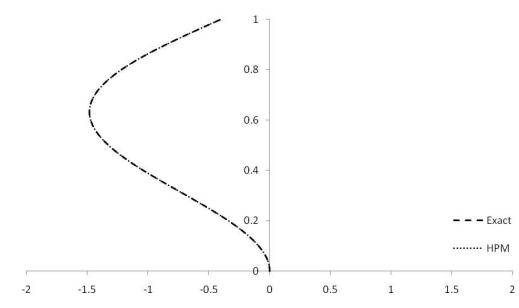

Figure 6. Mode shapes for the second mode - constant stiffness.

Polynomial approximations for exponential, linear and quadratic variations of flexural stiffness are normalized by using (6.1). Below, the first two mode shapes for different variations with previously defined parameters which depend on different values of $b$, are shown in Figs.7 - 12 .

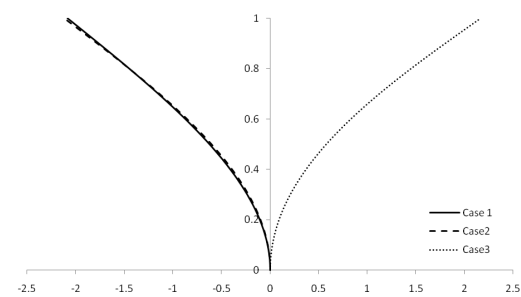

Figure 7. Modeshapes for the first mode - exponential variation.

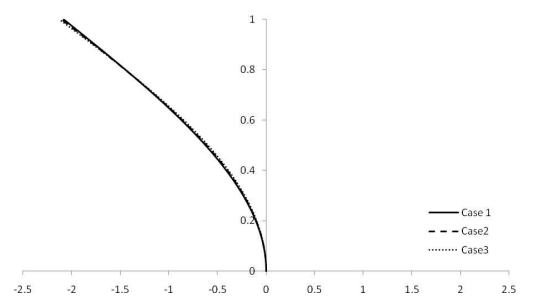

Figure 9. Modeshapes for the first mode - linear variation.

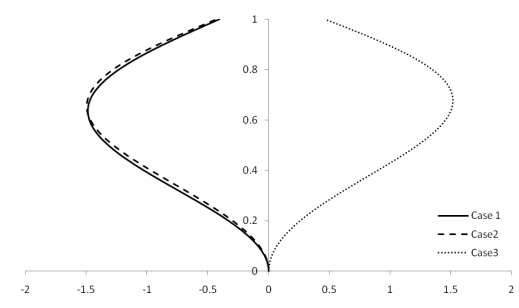

Figure 8. Modeshapes for the second mode - exponential variation.

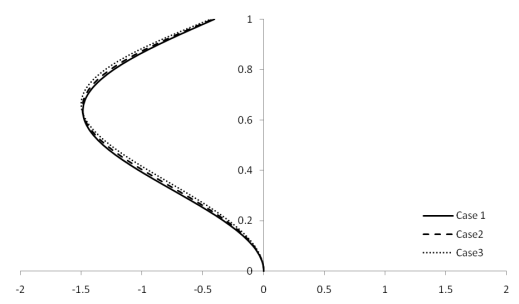

Figure 10. Modeshapes for the second mode - linear variation.

It may be observed that not only the buckling loads but also the buckling modeshapes can also be easily obtained in the elastic stability analysis of variable stiffness tilt-buckled columns. 


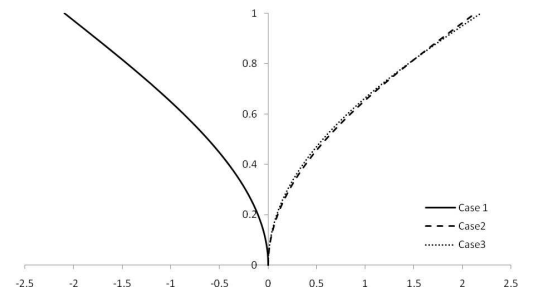

Figure 11. Modeshapes for the first mode - quadratic variation.

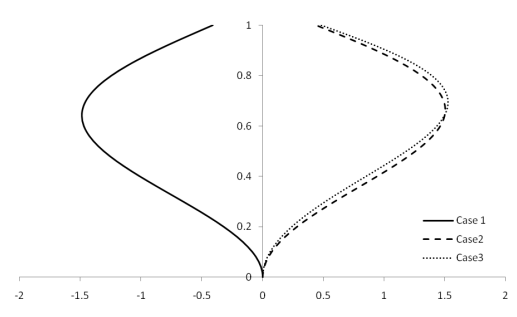

Figure 12. Modeshapes for the second mode - quadratic variation.

\section{Conclusion}

HPM was introduced in this study for the analysis of tilt-buckling of Euler columns with constant and variable flexural stiffness. As a demonstration of application of the method, first constant stiffness columns were analyzed and obtained results were compared with the exact ones. HPM results have shown excellent agreement with exact solutions for both the buckling loads and the mode shapes. The results for these cases are very accurate and show that the proposed method is capable to solve the same problems for columns of variable stiffness. By introducing a variable flexural stiffness for the buckling of an Euler column, it becomes difficult to obtain analytical solution for the problem. Each different assumption in the variation of flexural stiffness leads to a different variable coefficient ODE to be solved. In most of the cases, it is not possible to obtain analytical solutions. However, it is easy to insert these corresponding variable stiffness terms into HPM analysis procedure and the results can be obtained after performing some iterations with the method. The results obtained in this study pointed out that the proposed method is an efficient and powerful technique in the analysis of tilt-buckling problems of Euler columns with arbitrary variations of flexural stiffness.

\section{References}

[1] F. Arbabi and F. Li. Buckling of variable cross-section columns - integral equation approach. J. Struct. Eng.-ASCE, 117(8):2426-2441, 1991. Doi:10.1061/(ASCE)0733-9445(1991)117:8(2426).

[2] T.M. Atanackovic. Stability Theory of Elastic Rods. World Scientific Publishing Company, 1997. (Series on Stability Vibration)

[3] M.T. Atay. Determination of critical buckling loads for variable stiffness Euler columns using homotopy perturbation method. Int. Journal Nonlinear Sci. and Numer. Simulation, 10(2):199-206, 2009.

[4] M.T. Atay and S.B. Coşkun. Elastic stability of Euler columns with a continuous elastic restraint using variational iteration method. Computers and Mathematics with Applications, 58:2528-2534, 2009. Doi:10.1016/j.camwa.2009.03.051.

[5] J. Biazar and H. Ghazvini. Convergence of the homotopy perturbation method for partial differential equations. Nonlinear Analysis: Real World Applications, 10:2633-2640, 2009. Doi:10.1016/j.nonrwa.2008.07.002. 
[6] S.B. Coşkun. Determination of critical buckling loads for Euler columns of variable flexural stiffness with a continuous elastic restraint using homotopy perturbation method. Int. Journal Nonlinear Sci. and Numer. Simulation, 10(2):191197, 2009.

[7] S.B. Coşkun and M.T. Atay. Determination of critical buckling load for elastic columns of constant and variable cross-sections using variational iteration method. Computers and Mathematics with Applications, 58:2260-2266, 2009. Doi:10.1016/j.camwa.2009.03.072.

[8] A.N. Dinnik. Design of columns of varying cross-section. Trans. ASME, 51, 1929.

[9] J. C. Ermopoulos. Buckling of tapered bars under stepped axial loading. $J$. structural Eng. ASCE, 112(6):1346-1354, 1986.

Doi:10.1061/(ASCE)0733-9445(1986)112:6(1346).

[10] J.M. Gere and W.O. Carter. Critical buckling loads for tapered columns. J.Struct. Eng.- ASCE, 88:1-11, 1962.

[11] J.H. He. A coupling method of a homotopy technique and a perturbation technique for non-linear problems. Int. J. Nonlin. Mech., 35:37-43, 2000. Doi:10.1016/S0020-7462(98)00085-7.

[12] J.H. He. An elementary introduction to recently developed asymptotic methods and nanomechanics in textile engineering. International Journal of Modern Physics B, 22:3487-3578, 2008. Doi:10.1142/S0217979208048668.

[13] J.H. He. An elemantary introduction to the homotopy perturbation method. Computers and Mathematics with Applications, 57:410-412, 2009. Doi:10.1016/j.camwa.2008.06.003.

[14] T.R. Karman and M.A. Biot. Mathematical Methods in Engineering. McGraw Hill,NewYork, 1940.

[15] J.B. Keller. The shape of the strongest column. Archive for Rational Mechanics and Analysis, 5:275-285, 1960. Doi:10.1007/BF00252909.

[16] F. Kerekes and CL. Hulsbos. Elastic stability of the top chord of a three-span continuous pony truss bridge. Iowa Eng.: Expt. Sta. Bull., 1954.

[17] S.Y. Lee and Y.H. Kuo. Elastic stability of non-uniform columns. J.Sound, Vibration, 148(1):11-24, 1991. Doi:10.1016/0022-460X(91)90818-5.

[18] Q.S. Li. Exact solutions for buckling of non-uniform columns under axial concentrated and distributed loading. Eur. J. Mech. A/Solids, 20:485-500, 2001. Doi:10.1016/S0997-7538(01)01143-3.

[19] Q.S. Li, H. Cao and G. Li. Static and dynamic analysis of straight bars with variable cross-section. Comput. Struct., 59:1185-1191, 1996.

Doi:10.1016/0045-7949(95)00333-9.

[20] A. Morley. Critical loads for long tapering struts. Engineering, pp. 104-295, 1917.

[21] Iyengar NGR. Structural Stability of Columns and Plates. New York, John Wiley and Sons, 1988.

[22] Jr. J.H.B. Sampaio and J.R. Hundhausen. A mathematical model and analytical solution for buckling of inclined beam columns. Appl. Math. Model., 22:405-421, 1998. Doi:10.1016/S0307-904X(98)10014-8. 
[23] A.M. Siddiqui, A.R. Ansari, A. Ahmad and N. Ahmad. On Taylor's scraping problem and flow of a Sisko fluid. Math. Model. Anal., 14(4):515-529, 2009. Doi:10.3846/1392-6292.2009.14.515-529.

[24] A.M. Siddiqui, S. Irum and A.R. Ansari. Unsteady squeezing flow of a viscous MHD fluid between parallel plates, a solution using the homotopy perturbation method. Math. Model. Anal., 13(4):565-576, 2008. Doi:10.3846/1392-6292.2008.13.565-576.

[25] A. Siginer. Buckling of columns of variable flexural stiffness. J. Eng. Mech.ASCE, 118:543-640, 1992. Doi:10.1061/(ASCE)0733-9399(1992)118:3(640).

[26] G.J. Simitses and D.H. Hodges. Fundamentals of Structural Stability. ElsevierButterworth-Heinemann Publishing, 2005.

[27] I. Tadjbakhsh and J.B. Keller. Strongest columns and isoperimetric inequalities for eigenvalues. J. Appl. Mech. ASME, 29:159-164, 1962.

[28] J.E. Taylor. The strongest column - an energy approach. J. Appl. Mech. ASME, 34:486-487, 1967.

[29] S.P. Timoshenko and J.M. Gere. Theory of Elastic Stability. McGraw Hill, NewYork, 1961.

[30] H.H. Vaziri and Xie. J. Buckling of columns under variably distributed axial loads. Comput. Struct., 45(3):505-509, 1992.

[31] C.M. Wang, C.Y. Wang and J.N. Reddy. Exact Solutions for Buckling of Structural Members. CRC Press LLC, Florida, 2005. 